\title{
Fatal Strongyloidiasis Latent over 42 Years in the Antineoplastic Chemotherapy of a Case with Malignant Lymphoma
}

\author{
Toshiya SuzuKI, Nobuo NARA, Shuji MIYAKE, Yoshinobu EISHI*, \\ Etsuro SUGIYAMA**, and Nobuo AOKI
}

\begin{abstract}
A 66-year-old man with non-Hodgkin lymphoma died of strongyloides hyperinfection following antineoplastic chemotherapy. The patient had a military service in Micronesia in World War II where he was supposed to acquire strongyloidiasis. Here we stress the utility of bronchoscopic examination and importance of patients' lives in tropical climates in immunocompromised patients.
\end{abstract}

Key words: Strongyloides hyperinfection, Recurrent autoinfection, Immunocompromised patient, Cimetidine, Bronchoscopic examination.

Strongyloidiasis is an infection caused by Strongyloides stercoralis which has been reported to live exclusively in tropical or subtropical climates (1). Asymptomatic infection after acquisition persisted for long periods by recurrent autoinfection (2). On the other hand, parasitic infection are known to become virulent in immunocompromised patients. We warn you of the danger of strongyloides hyperinfection in immunocompromised patients.

\section{CASE REPORT}

A 66-year-old man was admitted to our hospital because of bilateral inguinal lymphnodes enlargement up to approximately $7 \times 4 \mathrm{~cm}$ in diameter on May 22, 1986. He was born and had lived in Tokyo. $\mathrm{He}$ had a military service in the Pagan Island, Micronesia, from 1944 to 1945 . The pathohistological diagnosis of the biopsied lymphnode was non-Hodgkin malignant lymphoma, diffuse large cell type (Working Formulation). The lymphoma cells were thought to be B cell lineage by surface marker analysis. HTLV-I and HTLV-III antibodies were negative. Physical and routine laboratory examination (lymphangiography, Ga scintigram, chest and abdominal echography) revealed that lymphoma cells were restricted to the bilateral inguinal region and the clinical stage was thought to be IIA. The patient received a combination chemotherapy; MACOP-B (methotrexate, adriamycin, cyclophosphamide, vincristine, prednisolone, and bleomycin) (3) on and after June 16. Cimetidine was medicated through the treatment. Since July 20, he had suffered from herpes zoster on his right leg. By the treatment with acyclovir, herpes zoster was improved. Transient eosinophilia was observed during the end of July to the beginning of August (348-616 cells/ $\mu 1)$. Neither asthma-like cough, skin rash, nor diarrhea was observed. Chest roentgenograms in August demonstrated coin-like lesion with cavity formation in the upper field of the right lung (Figure 1A). The coin-like lesion increased in size and amphotericin B was administered since August 13 because the lesion was suspected of aspergillus infection. To confirm the diagnosis of the lesion,

From The First Department of Internal Medicine, *The First Department of Pathology and **The Department of Parasitology, School of Medicine, Tokyo Medical and Dental University, Tokyo.

Received for publication June 21, 1988.

Reprint request to Toshiya Suzuki, MD, The First Department of Internal Medicine, Tokyo Medical and Dental University, 5-45, Yushima 1-Chome, Bunkyo-Ku, Tokyo 113, Japan. 


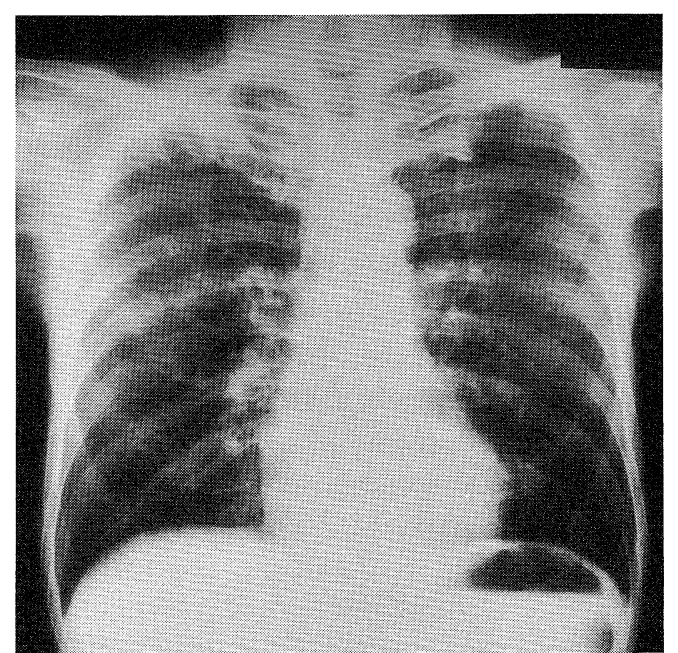

Fig. 1A. Coin like-lesion with cavity formation in the upper field of the right lung.

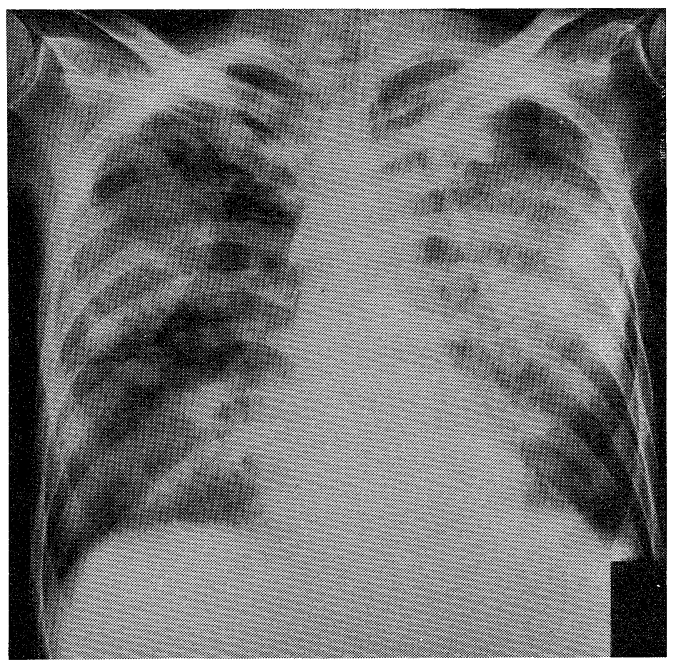

Fig. 1B. Diffuse intestinal and alveolar infiltration scattered over the bilateral lungs. Taken just before his death.

bronchoscopic examination was carried out on August 20. The bronchoscopic macro findings were negative. However, many filariform larvae were found in the bronchioalveolar lavage (BAL) (Figure 2A). Rhabditiform larvae were also found in the gastric juice. Under the diagnosis of strongyloidiasis, he received thiabendazole. After medication, the number of larvae in the sputa and gastric juice decreased markedly. Nevertheless viable larvae were noted in the following days. He suffered from Streptococcus faecalis bacteremia and died of

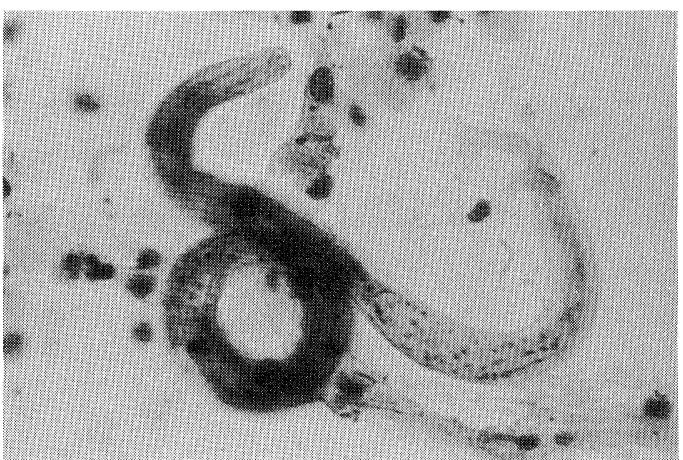

Fig. 2A. Filariform larva found in the BAL. (Papanicolaou's staining; original magnification, $\times 1000)$.

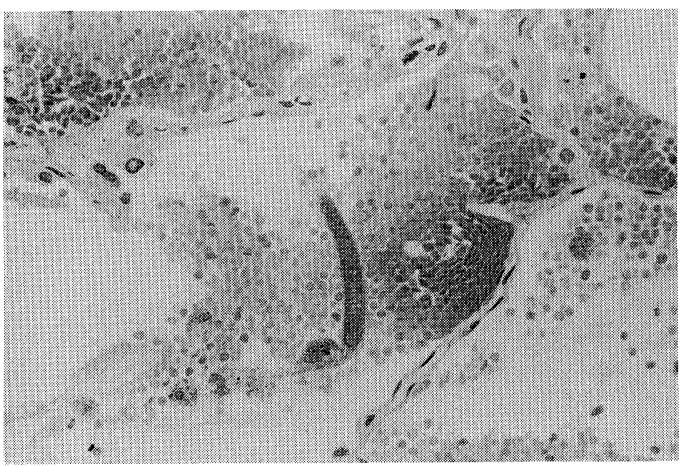

Fig. 2B. Larva in the alveolar space. Marked hemorrhage and a few macrophages are noted. (HE staining; original magnification, $\times 400$ ).



Fig. 2C. Eggs in the duodenum. (HE staining; original magnification, $\times 100$ ).

massive pulmonary and gastrointestinal hemorrhage on August 28 (Figure 1B).

Autopsy was performed with permission. Systemic hemorrhagic diathesis, which was considered to be the direct cause of his death was 


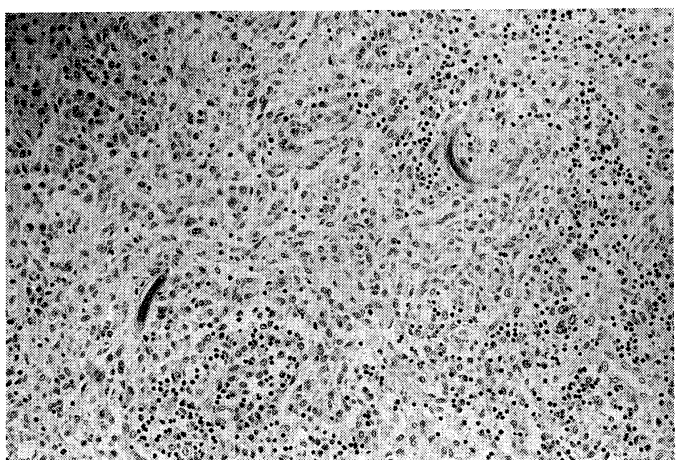

Fig. 2D. Larvae in the retroperitoneal lymph nodes. (HE staining; original magnification, $\times 200$ )

apparent; subarachnoidal, pulmonary, and gastrointestinal hemorrhage. Many larvae and eggs were found in the lungs, gastrointestinal tract, and retroperitoneal lymph node (Figure 2B, 2C, and 2D) while larvae were not detected in the other systemic organs. The coin-like lesion of the upper right lung was revealed to be aspergillus cavity. The inguinal lymph nodes were almost replaced by fibrous tissue and no abnormal lymphoma cells were noted. Bone marrow was significantly hypocellular one showing the markedly suppressed hematopoiesis.

\section{DISCUSSION}

Stronglyoidiasis is endemic in tropical and subtropical climates. In Japan, endemic regions are restricted; Amami-Oshima Island, Nansei Islands, Okinawa, and Kyushu (1). In many parts of Okinawa, $1-10 \%$ of residents were carriers of strongyloidiasis (4). Lymphoid malignancy, such as ATL or malignant lymphoma is often complicated by strongyloidiasis in Okinawa. Shiroma et al. (5) reported that they had 1016 cases of strongyloidiasis since 1953 to 1975 : 59 cases became hyperinfection and 24 cases of them were fatal. Outside of Okinawa, there were some reports of fatal strongyloidiasis complicated by ATL (6-8). All of the patients were born in endemic areas of the parasite.

In this case the patient was born and lived in Tokyo, non-endemic area of the parasite. Since he is considered to acquire strongyloidiasis at World War II, 42 years ago, the route of infection was considered as follows: The strongyloides filariform larvae (infective) burrowed through the skin when he walked about the tropical soil without shoes or invaded through gastrointestinal mucous when he drinked dirty water. The filariform larvae gained access to the blood stream and reached the lungs via right heart. The larvae climbed the bronchial tree and then descended into the gastrointestinal tract. Some of them became female (never male in human body) worms and lay eggs parthenogenetically in the mucous of duodenum. Others were released with stools. The eggs incubated to non-infective rhabditiform larvae. Some of them matured to filariform in the gastrointestinal tract and burrow through the mucous or skin again. This procedure is called autoinfection (2). In this patient, autoinfection was recurred after occult strongyloidiasis and persisted for 42 years. Strongyloidiasis that persisted for long periods after acquisition were also reported by others (9).

Cellular immunodeficiency caused by malignant lymphoma by itself and intensive antineoplastic chemotherapy was considered to allow the increment of the number of Strongyloides stercoralis explosively and killed the patient by pulmonary and gastrointestinal hemorrhage. The findings that no eosinophilia was noted at the hyperinfection state and that little inflammatory cells surrounded the parasites in the pathohistological specimens obtained from the autopsy suggest the immunodeficiency of the patients. He also suffered from herpes zoster and aspergillus infection; so called opportunistic infection. These opportunistic infection may also suggest the patients' immunodeficiency. The patient had received cimetidine, $\mathrm{H} 2$ receptor blockade, which is thought to reduce the defensive mechanism against strongyloidiasis by gastric juice (10).

The patient suffered from marked thrombocytopenia due to antineoplastic chemotherapy. The fatal hemorrhage from the respiratory and gastrointestinal tract might be caused by the active creeping of the larvae in these organs during the thrombocytopenia phase. Also marked granulocytopenia allowed Streptococcus faecalis bacteremia presumably caused by the migration of larvae from gastrointestinal tract to blood stream.

The case record of the patient may warn us to be careful to treat immunocompromised patients when they have the histories of military service or long-term lives in the tropical or subtropical 
climates. In this subpopulation, stools should be examined for strongyloides infection and if positive results are gained, they should be treated with thiabendazole as soon as possible until no strongyloides are found in the stools.

Finally, bronchoscopic examination was useful in the diagnosis of strongyloidiasis. The infection was diagnosed by the examination of the BAL specimens which were carried out to clarify aspergillus infection. Others also recommended the utility of bronchoscopic examination in the diagnosis of strongyloidiasis $(2,11)$. Many valuable data are provided by the bronchoscopic examination in the immunocompromised patients, so we should not be hesitated to perform bronchoscopic examination if there are some suspicious findings on the chest roentgenograms.

\section{REFERENCES}

1) Fujita K: Toxoplasmosis and strongyloidiasis as opportunistic infections. Biomedical and Therapeutics 13: 519, 1984 (in Japanese).

2) White JV, Garvey G, Hardy MA: Fatal strongyloidiasis after renal transplantation. Am Surgeon 48: 39, 1982.

3) Klimo P, Connors JM: MACOP-B chemotherapy for the treatment of diffuse large cell lymphoma. Ann Intern Med 102: 596, 1985.

4) Nakada K, Kohakura M, Komada H, et al: High incidence of HTLV antibody in carriers of Strongyloides stercoralis. Lancet i: 633, 1984.

5) Shiroma $Y$, Makina $T$, Uchihara $H$, et al: The study of the cases with strongyloides hyperinfection. Okinawa Medical Journal 13: 97, 1976 (in Japanese).

6) Matsui $T$, Ino $T$, Ono $Y$, et al: A case of adult $T$-cell leukemia with disseminated strongyloidiasis. Jpn J Clin Hematol 26: 1159, 1985 (in Japanese; abstract in English).

7) Terada A, Utsunomiya A, Matsumoto T, et al: Severe diarrhea and intestinal strongyloidiasis in a patient with adult T-cell leukemia. Acta Haematol Jpn 48: 1109, 1985.

8) Maekawa I, Kawamura T, Miyake T: Chronic adult Tcell leukemia (ATL) complicating disseminated strongyloidiasis. Jpn J Clin Hematol 29: 64, 1988 (in Japanese; abstract in English).

9) Pelletier LL: Chronic strongyloidiasis in World War II Far East ex-prisoners of war. Am J Trop Med Hyg 33: $55,1984$.

10) Ainley CC, Clarke DG, Timothy AR, et al: Strongyloides stercoralis hyperinfection associated with cimetidine in an immunocompromised patient. Gut 27: $337,1986$.

11) Smith B, Verghese A, Guiterrez C, et al: Pulmonary strongyloidiasis: Am J Med 79: 663, 1985. 